Boletín de la Sociedad Geológica Mexicana

VOLUMEN 61, NÚM. 2, 2009, P. 277-286

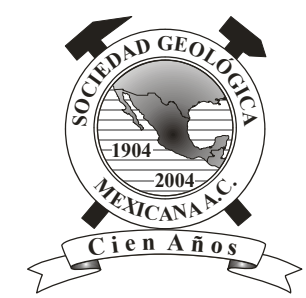

\title{
Contribución al conocimiento de los rinocerontes fósiles de la Cuenca de Tecolotlán, en el estado de Jalisco, México
}

\author{
Gerardo Carbot-Chanona ${ }^{1, *}$, Javier Juárez-Woo², José Rubén Guzmán-Gutiérrez ${ }^{3}$ \\ ${ }^{1}$ Museo de Paleontología “Eliseo Palacios Aguilera”, Instituto de Historia Natural. Calzada de Los Hombres Ilustres s/n, C. P. 29000. \\ Tuxtla Gutiérrez, Chiapas, México. \\ ${ }^{2}$ Museo de Paleontología de Guadalajara “Federico A. Solórzano Barreto”. Dr. R. Michel 520, C. P. 44100, Guadalajara, Jalisco, \\ México. \\ ${ }^{3}$ Departamento de Paleontología, Desarrollo Turístico El Caracol, SECTURE, Av. Perseo s/n. Aguascalientes, Aguascalientes, \\ México. \\ *carbotsaurus@yahoo.com
}

\section{Resumen}

Se describe el material de Teleoceras hicksi (Perissodactyla, Rhinocerotidae), depositado en el Museo de Paleontología de Guadalajara y en el Museo Regional de Guadalajara, proveniente de los sedimentos fluviolacustres de la Cuenca de Tecolotlán, Jalisco, México. Los restos examinados consisten en un cráneo incompleto con las series $\mathrm{P}^{2}-\mathrm{M}^{3}$ y una mandíbula asociada con ambos $\mathrm{I}_{2}$ y las series $\mathrm{P}_{3}-\mathrm{M}_{3}$, parte anterior de una mandíbula aislada con el $\mathrm{I}_{2}$ izquierdo y ambas series $\mathrm{P}_{3}-\mathrm{M}_{3}$, una mandíbula aislada con la serie $\mathrm{P}_{3}-\mathrm{M}_{3}$ pero sin sínfisis, molares superiores aislados, dos magno derechos, un metacarpo III izquierdo y la primera falange del digito III derecho. Se observaron variaciones morfológicas en el material examinado que refleja diferencias de edad y/o sexo entre los individuos. Teleoceras hicksi se encuentra en la Cuenca de Tecolotán asociado con Dinohippus mexicanus, Nannippus aztecus, Neohipparion eurystyle, Astrohippus stockii y Borophagus secundus, por lo que la edad de la fauna corresponde al Henfiliano tardío.

Palabras clave: Cuenca de Tecolotlán, Jalisco, Rhinocerotidae, Teleoceras hicksi, Henfiliano tardío.

\begin{abstract}
Remains of Teleoceras hicksi (Perissodactyla, Rhinocerotidae), housed at the "Museo de Paleontologia" in Guadalajara and the "Museo Regional de Guadalajara", recovered from the fuvial lacustrine sediments in the Tecolotlan Basin are described. The studied material includes a partial skull bearing $P^{2}-M^{3}$ and one lower jaw preserving $I_{2}$ and $P_{3}-M_{3}$, the anterior part of an isolated lower jaw with left $I_{2}$ and $P_{3}-M_{3}$, one isolated lower jaw with $P_{3}-M_{3}$ dental series in wich the symphyseal region is missing, also isolated molars, two magnus, one left metacarpus III, and one first phalange of the right digit III. Morphological differences were observed in the material examined attributed to differences in age and/or sex of the individuals. Teleoceras hicksi was found associated with Dinohippus mexicanus, Nannippus aztecus, Neohipparion eurystyle, Astrohippus stockii and Borophagus secundus, suggesting a late Hemphillian age for the faunal association.
\end{abstract}

Keywords: Tecolotlán Basin, Jalisco, Rhinocerotidae, Teleoceras hicksi, late Hemphillian. 


\section{Introducción}

Los rinocerontes fósiles son conocidos en México desde finales del siglo XIX, cuando E.D. Cope (1884) revisó los ejemplares fósiles de vertebrados del antiguo Museo Nacional de México, mencionando la mitad de la rama mandibular de Aphelops? fossiger (= Teleoceras fossiger) procedente del Valle de Toluca, Estado de México, la cual ahora está depositada en el Museo del Instituto de Geología de la Universidad Nacional Autónoma de México. A partir de ahí, se han registrado restos de Teleoceras en localidades miocénicas del norte, centro y sureste del país. Por ejemplo, T. fossiger ha sido reportado de localidades del Mioceno tardío de Michoacán (Brunet, 1968), Sinaloa (MaldonadoKöerdell, 1954) y Guanajuato (Carranza-Castañeda y Ferrusquía-Villafranca, 1978; Carranza-Castañeda, 1989), mientras que restos asignados a Teleoceras sp. provienen de localidades en Chihuahua, Jalisco, Guanajuato, Hidalgo, Morelos, Estado de México y Chiapas (Brunet, 1968; Lance, 1950; Lindsay, 1984; Ferrusquia-Villafranca, 1990; Castillo-Cerón et al., 1996; Miller y Carranza-Castañeda, 2002; Carbot-Chanona, 2008). Freudenberg $(1921,1922)$ incluyó a Teleoceras felicis como parte de la fauna de Tehuichila, Hidalgo, pero no mencionó el material en que basó su asignación y a la fecha se desconoce su paradero, haciendo imposible su revisión. Dalquest y Mooser (1980) describieron a $T$. ocotensis como una nueva especie para la Fauna Local Rancho El Ocote, con base en un $\mathrm{M}^{3}$, sin embargo Carranza-Castañeda (1989) consideró que se trata de un ejemplar joven y sinonimizó a esta especie con T. fossiger. Prothero (2005) a su vez sinonimizó a $T$. ocotensis con $T$. hicksi, y en consecuencia refirió todo el material de rinocerontes de la fauna Rancho El Ocote a esta especie. Es claro entonces, que a pesar de la extensa distribución geográfica de Teleoceras en México, muchos de los registros están basados en material fragmentario o pobremente conservado, lo que ha dificultado su asignación a nivel de especie; a excepción del material de rinocerontes proveniente de la Fauna del Rancho El Ocote, Guanajuato, el cual, hasta el momento es el mejor estudiado.

Los primeros trabajos sobre rinocerontes fósiles en el estado de Jalisco fueron hechos por Brunet (1968) quien reportó el género Teleoceras con base en un cráneo incompleto procedente de Tecolotlán (el mismo que se describe en este trabajo). Trabajos posteriores han mencionado la presencia de Teleoceras para la misma área (e.g. Rodríguez-Huerta y Guzmán-Gutiérrez, 1993; Miller y Carranza-Castañeda, 1998; Carranza-Castañeda, 2006), sin embargo, únicamente se menciona el material sin entrar en mayor detalle.

Según Brunet (1968), los ejemplares descritos en este trabajo fueron recolectados por pobladores de la región en los alrededores del poblado de Santa María, cerca de Tecolotlán, Jalisco, aproximadamente a $112 \mathrm{~km}$ al suroeste de la ciudad de Guadalajara (Figura 1) y posteriormente fueron donados a Federico Solórzano Barreto en el año 1960, quien depositó el material en el Museo de Paleontología de Guadalajara "Federico A. Solórzano Barreto" y en el Museo Regional de Guadalajara. Estudios posteriores señalan que todo el material de rinocerontes proviene de los sedimentos fluviolacustres de la Cuenca de Tecolotlán.

\section{Geología y paleontología de la Cuenca de Tecolotlán}

La Cuenca de Tecolotlán se sitúa en la Sierra Madre Occidental dentro de la Faja Volcánica Transmexicana, a $100 \mathrm{~km}$ al suroeste de Guadalajara; tiene un área de $18 \mathrm{~km}$ por $10 \mathrm{~km}$ y se orienta de norte a sur (Miller y CarranzaCastañeda, 1998). Carranza-Castañeda (2006) describió la secuencia fluviolacustre de la Cuenca de Tecolotlán, diferenciando dos secciones que en total miden alrededor de $80 \mathrm{~m}$ (Figura 2). La sección inferior de la secuencia está compuesta por estratos de arcilla, arena arcillosa finogranuda y caliche; en la parte superior está presente una capa de ceniza volcánica que ha sido datada mediante ${ }^{40} \mathrm{Ar} /{ }^{39} \mathrm{Ar}$ en una edad de $4.89 \pm 0.016 \mathrm{Ma}$ (Kowallis et al., 1998 en Carranza-Castañeda, 2006). De esta sección se han recuperado restos de Crocodylus sp., Gopherus sp., Notolagus velox, Hypolagus sp., Spermophilus sp., Calomys sp., Agriotherium schneideri, Pseudaelurus cf. $P$. intrepidus, Machairodus cf. M. coloradensis, Canis ferox, Astrohippus stockii, Nannippus aztecus, Neohipparion eurystyle, Dinohippus mexicanus, Rhyncotherium $\mathrm{cf} . R$. falconeri, Hemiauchenia vera, Megalonyx sp., Megatylopus cf. M. matthewi, Borophagus secundus (=Osteoborus cyonoides) y Teleoceras hicksi. La edad de la sección ha sido determinada como Henfiliano tardío con base en la asociación faunística (Miller y Carranza-Castañeda, 1998; Carranza-Castañeda, 2006) y puede correlacionarse con las localidades de Yepómera, Chihuahua (Lance, 1950; Lindsay, 1984), El Ocote y Rinconada, Guanajuato (CarranzaCastañeda y Ferrusquía-Villafranca, 1978; Dalquest y Mooser, 1980; Carranza-Castañeda, 1992) y Teocaltiche, Jalisco (Montellano-Ballesteros, 1997).

La sección superior de la secuencia está conformada por estratos de arena finogranuda y grava en la que también se observa una capa de ceniza volcánica datada en $2.6 \pm 0.2 \mathrm{Ma}$ con el método ${ }^{40} \mathrm{Ar} /{ }^{39} \mathrm{Ar}$ (Kowallis et al., 1998 en CarranzaCastañeda, 2006). En esta sección se han recuperado restos de Glyptotherium sp., Neochoerus sp., Platygonus sp., cf. Plaina y Equus simplicidens que indican Blancano más tardío; sobre está parte se ubican discordantemente estratos conformados por arcilla y grava de edad reciente (CarranzaCastañeda, 2006).

\section{Material y método}

El material descrito en este trabajo fue comparado con el estudiado por Carranza-Castañeda (1989) proveniente de la fauna local Rancho El Ocote, Guanajuato, determinado 


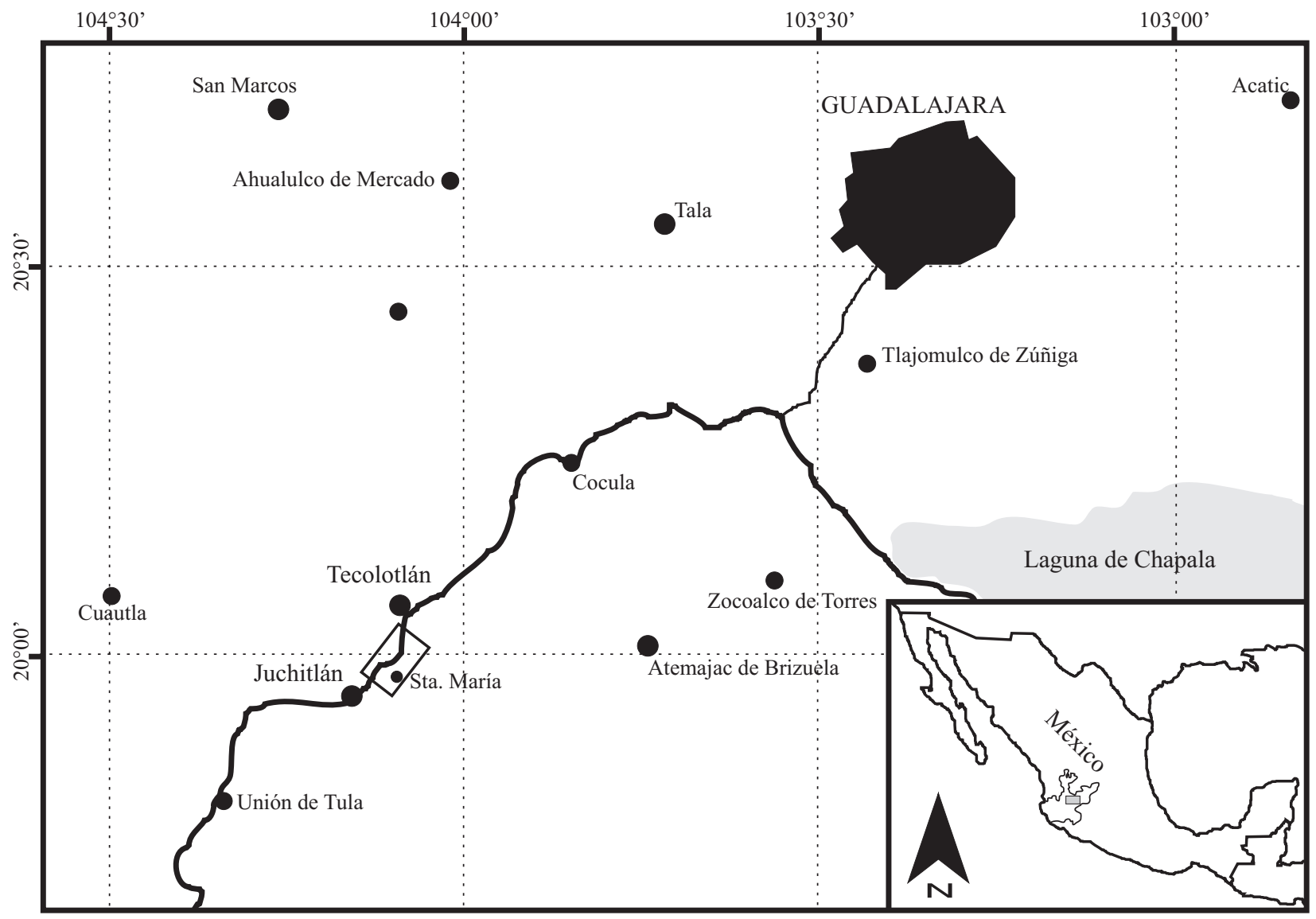

Figura 1.- Localización de la localidad fosilífera en el área de Tecoltlán-Juchitlán, Jalisco.

como Teleoceras fossiger $(=T$. hicksi), así como con las descripciones e ilustraciones presentadas en los trabajos de Hatcher (1894), Osborn (1904) y Prothero (2005). La nomenclatura morfológica utilizada para la descripción está basada en Prothero (2005).

Las medidas se obtuvieron tomando la longitud y anchura de la superficie oclusal de molares y premolares superiores e inferiores que presentan distintos grados de desgaste. En el caso del cráneo y las mandíbulas se tomaron diferentes medidas, las cuales se detallan en las Tablas 1 y 2. Se describen cuatro elementos apendiculares y sus dimensiones se muestran en la Tabla 4. Todas las medidas se expresan en milímetros.

Las abreviaturas y siglas utilizadas en este trabajo son: I, incisivo; IGM, Instituto Geológico de México; $\mathrm{P}^{\mathrm{X}}$, premolar superior; $\mathrm{P}_{\mathrm{X}}$, premolar inferior; $\mathrm{M}^{\mathrm{X}}$, molar superior; $\mathrm{M}_{\mathrm{x}}$, molar inferior; mm, milímetros; MPG-R, Museo de Paleontología de Guadalajara-Referencia; MRG, Museo Regional de Guadalajara; UNAM, Universidad Nacional Autónoma de México.

\section{Sistemática Paleontológica}

Orden Perissodactyla Owen, 1848
Familia Rhinocerotidae Gray, 1821

Tribu Teleoceratini Hay, 1902

Género Teleoceras Hatcher, 1894

Teleoceras hicksi Cook, 1927

Especie tipo. Teleoceras major Hatcher, 1894. Clarendoniano temprano, Nebraska

Sinonimias. Paraphelops yumensis (Cook, 1930), Paraceras ponderensis (Cook, 1930), Teleoceras yumensis (Matthew, 1932), Teleoceras schultzi (Tanner, 1975), Teleoceras ocotensis (Dalquest y Mooser, 1980), Teleoceras fossiger (Carranza-Castañeda, 1989).

Diagnosis. Teleoceras de mediano tamaño (longitud del $\mathrm{M}^{1}-\mathrm{M}^{3}=150-165 \mathrm{~mm}$, longitud del $\mathrm{M}_{1}-\mathrm{M}_{3}=155-180 \mathrm{~mm}$ ) con cráneo más braquicefálico que $T$. fossiger. Nasales cortos y ligeramente levantados en muchos especímenes, pero no aplanados como en T. brachyrhinum. $\mathrm{P}_{2}$ ausente (Prothero, 2005).

\subsection{Material referido}

MRG-10-295030 cráneo (parte A) y mandíbula (parte B); MPG-R-0137, mandíbula con las series $\mathrm{P}_{3}-\mathrm{M}_{3}$ derecha e izquierda, pero sin la sínfisis; MPG-R-0248, parte anterior de la mandíbula con los $\mathrm{I}_{2}$, y las series $\mathrm{P}_{3}{ }^{-}$ 
Tabla 1. Medidas (en mm) comparativas del cráneo de Teleoceras hicksi de la Cuenca de Tecolotlán, Jalisco y de la fauna local Rancho El Ocote, Guanajuato. e = en erupción.

\begin{tabular}{|c|c|c|c|c|c|}
\hline & & MRG-10-295030-A & IGM-8357 & IGM-8358 & IGM-8360 \\
\hline \multicolumn{2}{|r|}{ Longitud máxima desde los cóndilos hasta el extremo anterior de $\mathrm{P}^{4}$} & - & 392 & 420 & 445 \\
\hline \multicolumn{2}{|r|}{ Anchura máxima entre las protuberancias orbítales } & 183 & 176 & - & - \\
\hline \multicolumn{2}{|r|}{ Anchura máxima entre los arcos zigomáticos en la porción anterior } & 250 & 258 & - & 292 \\
\hline \multicolumn{2}{|r|}{ Longitud máxima entre los arcos zigomáticos en el extremo posterior } & - & 347 & - & 310 \\
\hline \multicolumn{2}{|r|}{ Altura máxima de la parte media de los cóndilos hacia la cresta sagital } & - & - & - & 133 \\
\hline \multicolumn{2}{|r|}{ Anchura máxima de la región occipital a través del conducto auditivo } & - & 246 & - & 260 \\
\hline \multicolumn{2}{|r|}{ Anchura máxima del paladar desde el borde interno del protocono de $\mathrm{M}^{2}$} & 70 & - & - & - \\
\hline \multicolumn{2}{|r|}{ Longitud máxima de la serie $\mathrm{M}^{3}-\mathrm{P}^{4}$ a través de la línea media } & 178 & 191 & 212 & 194 \\
\hline & Longitud & 47 & $\mathrm{e} 27$ & 64 & 54 \\
\hline & Anchura & 51 & e26 & 51 & 44 \\
\hline & Longitud & 51 & 60 & 59 & 57 \\
\hline & Anchura & 65 & 34 & 63 & 55 \\
\hline & Longitud & 42 & 64 & 49 & 43 \\
\hline & Anchura & 59 & 44 & 64 & 59 \\
\hline & Longitud & 38 & 40 & 40 & 40 \\
\hline & Anchura & 54 & 35 & 57 & 50 \\
\hline & Longitud & 32 & - & 35 & - \\
\hline & Anchura & 44 & - & 47 & - \\
\hline & Longitud & 29 & - & - & - \\
\hline & Anchura & 32 & - & - & - \\
\hline
\end{tabular}

Tabla 2. Medidas (en mm) de las mandíbulas de Teleoceras hicksi de la Cuenca de Tecolotlán (Jalisco) y de la fauna local Rancho El Ocote (Guanajuato). $\mathrm{d}=$ deciduo.

\begin{tabular}{|c|c|c|c|c|c|c|}
\hline & & MRG-10-295030-B & MPG-R-0137 & MPG-R-0248 & IGM-8361 & IGM-8362 \\
\hline \multicolumn{2}{|r|}{ Longitud máxima desde el cóndilo hasta el extremo anterior de $\mathrm{I}_{2}$} & 440 & - & - & 662 & - \\
\hline \multicolumn{2}{|r|}{ Longitud máxima desde el cóndilo hasta la cara anterior de $\mathrm{P}_{3}$} & 330 & 380 & - & 437 & 315 \\
\hline \multicolumn{2}{|r|}{ Anchura máxima transversal de la sínfisis } & 120 & - & 106 & 106 & - \\
\hline \multicolumn{2}{|r|}{ Altura de la mandíbula desde el cóndilo hasta el ángulo mandibular } & 210 & 200 & - & - & 215 \\
\hline \multicolumn{2}{|r|}{ Altura de la mandíbula a través del foramen mentoniano } & 73 & 63 & 89 & - & 67 \\
\hline & Longitud & 50 & 52 & 54.5 & 60 & - \\
\hline & Anchura & 28 & 27 & 24 & 30 & - \\
\hline & Longitud & 48 & 51 & 50 & 55 & 50 \\
\hline & Anchura & 31 & 30 & 26 & 35 & 21 \\
\hline & Longitud & 42 & 43 & 43 & 46 & 44 \\
\hline & Anchura & 30 & 32 & 26 & 36 & 22 \\
\hline & Anchura & 27 & 23 & 26 & 31 & $22 \mathrm{~d}$ \\
\hline \multirow{2}{*}{$\mathrm{P}_{3}$} & Longitud & 30 & 31 & 32 & 30 & $20 d$ \\
\hline & Anchura & 22 & 20 & 21 & 24 & $14 d$ \\
\hline & Diámetro anteroposterior & 31 & - & 31 & 34 & - \\
\hline & Diámetro transversal & 50 & - & 44 & 39 & - \\
\hline
\end{tabular}

$\mathrm{M}_{3}$ derecha e izquierda; MPG-R-3200, $\mathrm{M}^{2}$ izquierdo; MPG-R-3201, $\mathrm{M}^{2}$ derecho; MPG-R-3202, $\mathrm{M}^{3}$ izquierdo; MPG-R-3203, $\mathrm{M}^{2}$ derecho; MPG-R-3204, $\mathrm{M}^{2}$ derecho; MPG-R-3205, $\mathrm{M}^{3}$ derecho; MPG-R-3207, $\mathrm{M}^{3}$ izquierdo; MPG-R-3208, fragmento del maxilar con el $\mathrm{P}^{2}-\mathrm{M}^{1}$ derechos; MPG-R-3209, fragmento del maxilar con el $\mathrm{M}^{1}$ y $\mathrm{M}^{2}$ izquierdos; MPG-R-3210, fragmento del maxilar con $\mathrm{M}^{2}$ y $\mathrm{M}^{3}$ izquierdos; MPG-R-3212, metacarpo III izquierdo; MPG-R-3213, primera falange del dígito III; MPG-R-3214, magno derecho; MPG-R-3215, magno derecho.

\subsection{Descripción del material}

MRG-10-295030-A consiste en la parte anterior de un cráneo bien conservado (Figura 3), en el cual toda la región occipital y los parietales no están presentes. Los arcos zigomáticos comienzan al nivel de $\mathrm{M}^{1}$, les falta la porción posterior, pero aun así puede apreciarse que son 


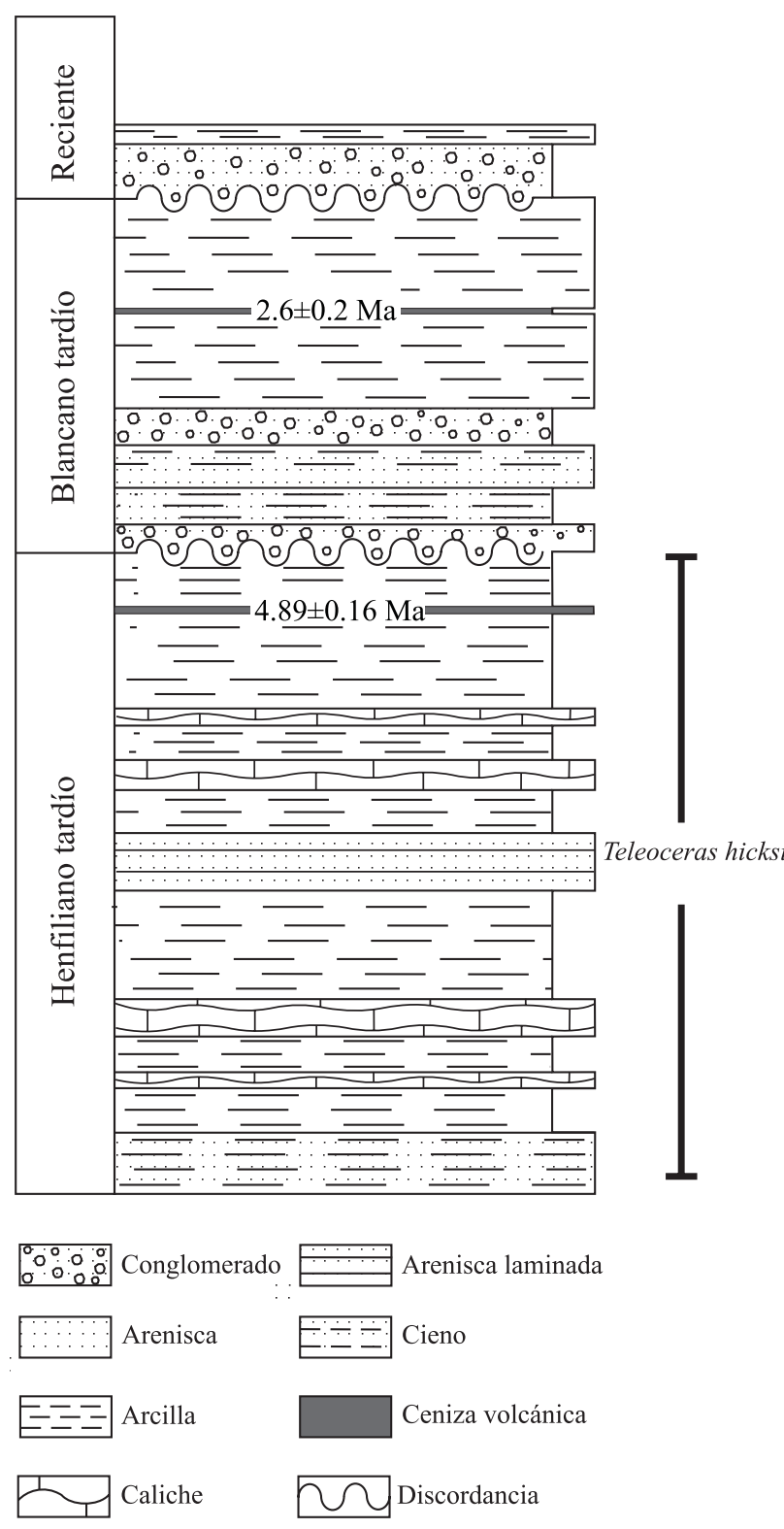

Figura 2.- Secuencia estratigráfica compuesta de la Cuenca de Tecolotlán. Modificado de Carranza-Castañeda (2006).

más estrechos anterior que posteriormente. Por encima de las órbitas se observan los procesos supraoccipitales bien desarrollados, lo que hace que los huesos frontales sean anchos y que se estrechen poco posteriormente. Premaxilares no fusionados, más largos que los nasales y conservan los alveolos de los $\mathrm{I}^{1}$. Los nasales son anchos pero se estrechan mucho hacia la parte anterior; los bordes laterales son redondeados, lo que hace que en sección transversal tengan forma de "U". Los molariformes están medianamente desgastados y se conservan las dos series dentarias completas, desde el $\mathrm{P}^{2}$ al $\mathrm{M}^{3}$, los cuales se describen individualmente a continuación.

$\mathrm{P}^{2}$.- Premolar ligeramente desgastado. La prefoseta es alargada y está constreñida sobre su parte media; postfoseta muy pequeña y de forma lenticular. El crochet y el anticrochet no se observan, posiblemente por el desgaste que presenta el diente. Valle medio cerrado por la fusión del metalofo y el protolofo. Cíngulo no presente. Parastilo reducido.

$\mathrm{P}^{3}$.- Premolar semicuadrado, con el parastilo muy pequeño; metalofo y protolofo fuertemente fusionados, cerrando el valle y formando la prefoseta, la cual es de mediano tamaño y alargada; postfoseta apenas visible. Crochet lobular y pequeño, anticrochet no visible. Cíngulo ligeramente visible en la pared del ectolofo.

$\mathrm{P}^{4}$.- Parastilo pequeño, ligeramente más proyectada labialmente que en el $\mathrm{P}^{3}$; protocono semicuadrado y ancho, ectolofo plano. Prefoseta alargada, postfoseta pequeña. Crochet muy pequeño, anticrochet no visible debido al desgaste del diente. Protolofo en contacto con el metalofo. Cíngulo lingual bien desarrollado.

$\mathrm{M}^{1}$.- Ectolofo ancho y convexo, parastilo medianamente desarrollado. Prefoseta alargada y abierta; postfoseta pequeña, constreñida en su parte media por el desarrollo del protolofo. Crochet no presente; anticrochet pequeño y casi en contacto con el protolofo. Parastilo medianamente desarrollado; cíngulo lingual apenas visible.

$\mathrm{M}^{2}$.- Prefoseta alargada y abierta sobre la cara lingual, postfoseta grande y de forma lenticular. Crochet redondeado y pequeño; anticrochet ancho y de forma lobular, de mayor tamaño que el crochet. Cíngulo visible por las caras anterior y posterior del diente. Metalofo y protolofo bien desarrollados.

$\mathrm{M}^{3}$.- Molar con el parastilo pequeño; protocono bien desarrollado. Prefoseta y mediofoseta constreñidas. Anticrochet muy pequeño, crochet más grande que el anticrochet, dirigido hacia él sin llegar a contactarlo. Cíngulo visible en el borde lingual del protolofo. Crista presente.

MRG-10-295030-B es una mandíbula en buen estado de preservación que conserva los dos $\mathrm{I}_{2} \mathrm{y}$ las dos series dentarias $\mathrm{P}_{3}-\mathrm{M}_{3}$ (Figura 4a). Los cóndilos articulares están completos; los procesos coronoides son rectos y orientados ligeramente hacia delante, rasgo característico de los teleoceratinos. El diastema es corto; las series dentarias son paralelas pero las ramas mandibulares están en $\mathrm{V}$ cerrada. La sínfisis es ancha; los $\mathrm{I}_{2}$ son anchos, dirigidos lateralmente y en sección transversal son semiovalados. $\mathrm{El} \mathrm{M}_{3}$ presenta un ligero cíngulo visible sólo por su parte labial. El foramen mentoniano está presente a la altura de la raíz del $\mathrm{P}_{4}$.

La morfología del cráneo y la mandíbula, el desgaste dental y la anchura del $\mathrm{I}_{2}$, indican que este ejemplar fue un macho adulto bien desarrollado.

MPG-R-0248. Mandíbula a la que le falta la mitad posterior de ambas ramas mandibulares, conservándose la serie $\mathrm{P}_{3}-\mathrm{M}_{3}$ derecha, y $\mathrm{P}_{4}-\mathrm{M}_{3}$ izquierda, así como el $\mathrm{I}_{2}$ izquierdo (Figura $4 b$ ). Las ramas mandibulares son más robustas que en MPG-R-0137 y el $\mathrm{I}_{2}$ es ligeramente más angosto transversalmente que el $\mathrm{I}_{2}$ de MRG-10-295030-B (Tabla 2); estas características indican que se trata de un 

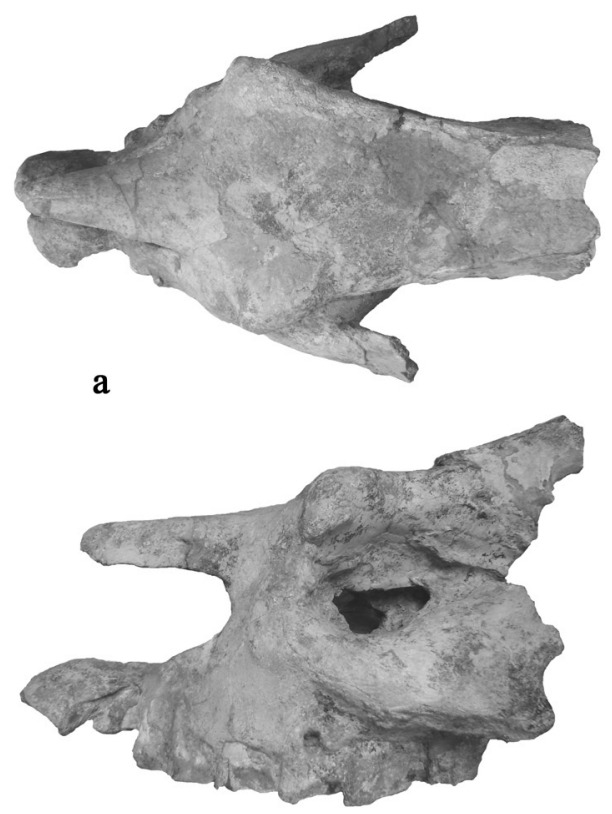

b

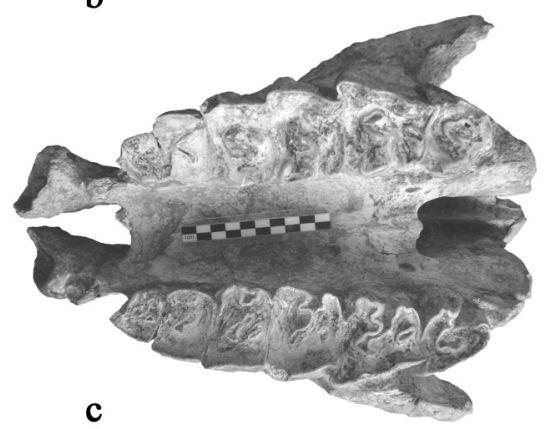

Figura 3.- Cráneo de Teleoceras hicksi de la Cuenca de Tecolotlán, Jalisco: MRG-10-295030-A, a) vista dorsal, b) vista lateral, c) vista oclusal. Barra de escala igual a $10 \mathrm{~cm}$.

macho adulto.

MPG-R-0137. Mandíbula casi completa a la cual le falta la sínfisis, del $\mathrm{P}_{3}$ en adelante, por lo que ambos $\mathrm{I}_{2}$ no están presentes (Figura 4c). A los procesos coronoides les falta la parte más proximal, sin embargo los cóndilos articulares están completos; el foramen mentoniano se encuentra ubicado ligeramente más abajo del comienzo de la raíz del $\mathrm{P}_{4}$. La dentición está conformada por $\mathrm{P}_{3}$ al $\mathrm{M}_{3}$ derechos e izquierdos, faltando únicamente la mitad anterior del $\mathrm{M}_{1}$ derecho. Las ramas mandibulares son ligeramente más gráciles que en MRG-10-295030-B y que en MPG-R-0248, lo que sugiere que se trata de un individuo juvenil.

\subsubsection{Molariformes aislados}

MPG-R-3209 es un fragmento del maxilar con el $\mathrm{M}^{1} \mathrm{y}$ el $\mathrm{M}^{2}$ derechos (Figura 5a), de los cuales el segundo está muy fragmentado. $\mathrm{M}^{1}$ con un grado de desgaste mínimo, lo que sugiere que el individuo era juvenil. Parastilo bien desarrollado; la prefoseta es profunda, alargada y se
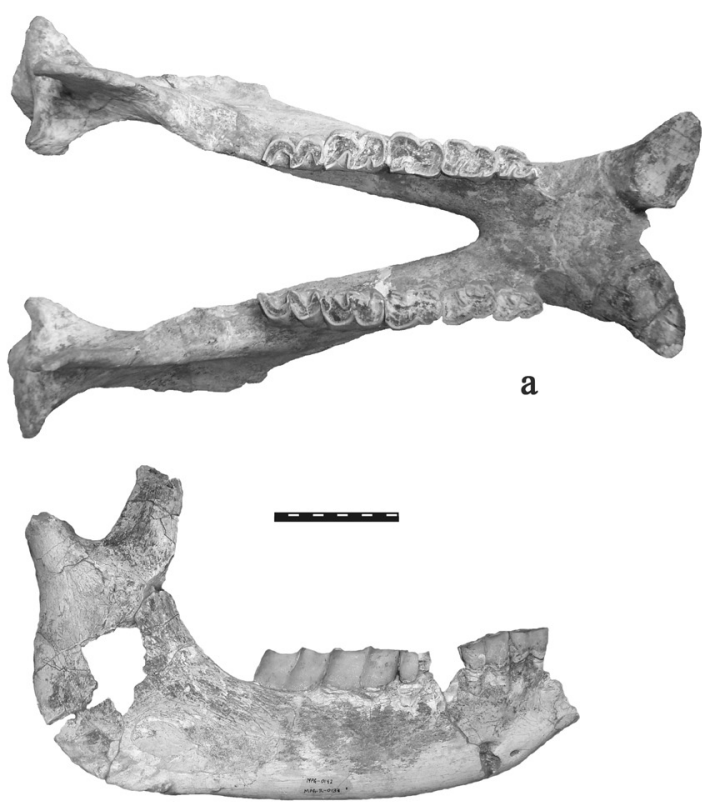

b

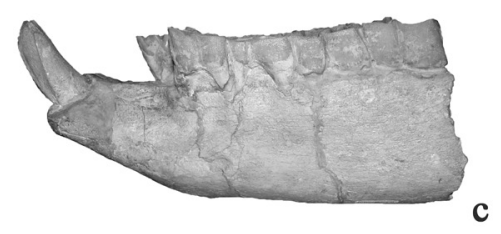

Figura 4.- Mandíbulas de Teleoceras hicksi de la Cuenca de Tecolotlán, Jalisco: a) MRG-10-295030-B, vista oclusal; b) MPG-R-0137, vista lateral; c) MPG-R-0248, vista lateral. Barra de escala igual a $10 \mathrm{~cm}$.

encuentra abierta; postfoseta grande. Protolofo y protocono constreñidos. Metalofo angosto. El cíngulo es fuerte en toda la cara lingual. Ectolofo ancho y convexo, con una altura de $53.5 \mathrm{~mm}$. Crochet pequeño; anticrochet ligeramente mayor pero más delgado. Crista reducida.

MPG-R-3200. $\mathrm{M}^{2}$ izquierdo con la prefoseta abierta; postfoseta pequeña. El crochet es de forma lobular, el anticrochet de forma similar pero ligeramente de mayor tamaño. El ectolofo es alto. Parastilo prominente. Metastilo, metacono y borde lingual del metalofo rotos. Hay cíngulos anterior y posterior (Figura $5 b$ ).

MPG-R-3201. $\mathrm{M}^{2}$ derecho con ectolofo reducido; protolofo prolongado. Protocono de forma semicuadrada por su parte lingual, con el esmalte formando una depresión. Parastilo y metastilo rotos. El cíngulo es visible en la parte anterior, posterior y lingual del diente. La postfoseta no se observa por el desgaste, pero la prefoseta es alargada. Crochet no visible por el desgaste del diente; anticrochet grande y de forma lobular (Figura 5c). 


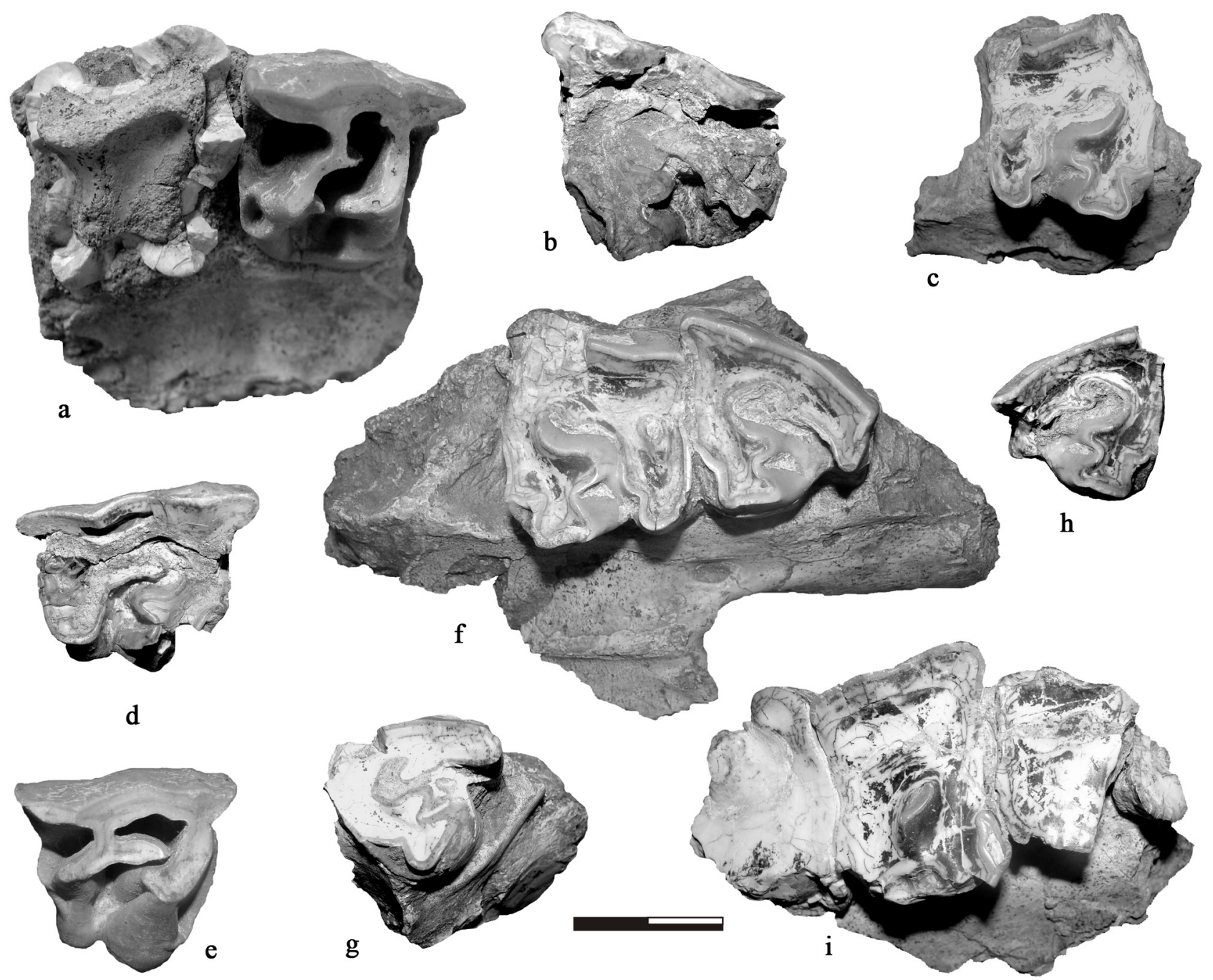

Figura 5.- Molares aislados de Teleoceras hicksi provenientes de la Cuenca de Tecolotlán, Jalisco: a) MPG-R-3209, $\mathrm{M}^{1}$-M² derechos; b) MPG-R-3200, $\mathrm{M}^{2}$ izquierdo; c) MPG-R-3201, $\mathrm{M}^{2}$ derecho; d) MPG-R-3203, $\mathrm{M}^{2}$ derecho; e) MPG-R-3204, $\mathrm{M}^{2}$ derecho; f) MPG-R-3210, fragmento del maxilar con $\mathrm{M}^{2}$ y $\mathrm{M}^{3}$ izquierdos; g) MPG-R-3202, $\mathrm{M}^{3}$ izquierdo; h) MPG-R-3205, $\mathrm{M}^{3}$ derecho; i) MPG-R-3208, $\mathrm{P}^{2}-\mathrm{M}^{1}$ derechos. Barra de escala igual a $4 \mathrm{~cm}$.

MPG-R-3203 es un $\mathrm{M}^{2}$ derecho, en el cual el borde lingual del protocono está fracturado. El parastilo es prominente y de forma lobular. Metastilo fracturado. El crochet es más pequeño que el anticrochet. Prefoseta y mediofoseta constreñidas. Postfoseta pequeña y cerrada. Borde del metalofo en forma de "U". Cíngulo no visible por la mala conservación del ejemplar (Figura 5d).

MPG-R-3204. $\mathrm{M}^{2}$ derecho con poco desgaste, que indica se trata de un animal joven. El ectolofo es ancho y convexo y tiene una atura de $75.2 \mathrm{~mm}$. Prefoseta profunda y abierta, ya que el valle no se ha fusionado. Postfoseta amplia. Crochet lobular y grande, anticrochet pequeño. Metalofo delgado y alargado El cíngulo no se observa (Figura 5e).

MPG-R-3210. Fragmento del maxilar que conserva el $\mathrm{M}^{2}$ y $\mathrm{M}^{3}$ izquierdos (Figura $5 \mathrm{f}$ ); ambos molariformes presentan un desgaste marcado, lo que sugiere que pertenecieron a un animal adulto. En el $\mathrm{M}^{2}$ el parastilo es grande y de forma lobular. El crochet no es visible, contrariamente al anticrochet que es grande y ancho y casi contacta al metalofo, lo que hace que la prefoseta presente un estrechamiento en esa zona. La postfoseta es muy pequeña. El valle se encuentra abierto. $\mathrm{El} \mathrm{M}^{3}$ tiene un protocono bien desarrollado. Parastilo y paracono pequeño. Metastilo prominente. Crochet muy reducido, debido tal vez al desgaste; anticrochet pequeño y de forma lobular. El valle medio está abierto desde la base. Ectolofo delgado. Cíngulo visible en todo el perímetro del molar.

MPG-R-3202. $\mathrm{M}^{3}$ izquierdo muy desgastado adherido a una porción del maxilar (Figura $5 \mathrm{~g}$ ). La prefoseta y postfoseta son profundas. El cíngulo es visible sólo en la parte lingual. Crochet y anticrochet de forma lobular y casi del mismo tamaño. Parastilo roto. Metastilo prominente. Protocono ancho.

MPG-R-3205 es un $\mathrm{M}^{3}$ derecho con el protocono bien 
Tabla 3. Medidas (en $\mathrm{mm}$ ) de molares superiores aislados de Teleoceras hicksi de la Cuenca de Tecolotlán, Jalisco. $(\approx)$ Medidas aproximadas.

\begin{tabular}{llcc}
\hline & & Longitud & Anchura \\
\hline MPG-R-3201 & $\left(\mathrm{M}^{2}\right)$ & $\approx 50$ & 50 \\
MPG-R-3200 & $\left(\mathrm{M}^{2}\right)$ & 62 & 58 \\
MPG-R-3203 & $\left(\mathrm{M}^{2}\right)$ & 65 & 46 \\
MPG-R-3204 & $\left(\mathrm{M}^{2}\right)$ & 59 & 55 \\
MPG-R-3205 & $\left(\mathrm{M}^{3}\right)$ & $\approx 48$ & 43 \\
MPG-R-3202 & $\left(\mathrm{M}^{3}\right)$ & $\approx 45$ & 40 \\
MPG-R-3207 & $\left(\mathrm{M}^{3}\right)$ & 54 & 44 \\
MPG-R-3209 & $\left(\mathrm{M}^{1}\right)$ & 44 & 63 \\
& $\left(\mathrm{M}^{2}\right)$ & - & - \\
MPG-R-3210 & $\left(\mathrm{M}^{2}\right)$ & 51 & 62 \\
& $\left(\mathrm{M}^{3}\right)$ & 47 & 45 \\
\hline
\end{tabular}

Tabla 4. Medidas (en $\mathrm{mm}$ ) de los huesos apendiculares de Teleoceras hicksi de la Cuenca de Tecolotlán, Jalisco. Abreviaturas: LAP, longitud anteroposterior; LT, longitud total; HA, altura anterior; HP, altura posterior; WD, ancho distal; WI, ancho en la parte media; WP, ancho proximal.

\begin{tabular}{lccccccc}
\hline & LT & WP & WD & WI & LAP & HP & HA \\
\hline $\begin{array}{c}\text { MPG-R-3212 } \\
\text { metacarpo III }\end{array}$ & 113 & 65 & 57 & 50 & - & - & - \\
$\begin{array}{c}\text { MPG-R-3213 } \\
\text { segunda falange }\end{array}$ & 58 & 59 & 52 & 43 & - & - & - \\
$\begin{array}{c}\text { MPG-R-3214 } \\
\text { magno derecho }\end{array}$ & - & - & - & - & 45 & 34 & 25 \\
$\begin{array}{c}\text { MPG-R-3215 } \\
\text { magno derecho }\end{array}$ & - & - & - & - & 49 & 35 & 27 \\
\hline
\end{tabular}

desarrollado; parastilo y metastilo rotos. Prefoseta pequeña; postfoseta no visible debido a que esa parte del diente está perdida. Crochet pequeño, anticrochet de mayor tamaño. Cíngulo pequeño en el borde lingual del protolofo (Figura 5h).

MPG-R-3207. $\mathrm{M}^{3}$ izquierdo con ectolofo semiplano. El parastilo, al igual que el protocono, bien desarrollados. Metalofo y protolofo unidos. Cíngulo visible en toda la pared del ectolofo, metalofo y protolofo. Prefoseta y postfoseta unidas. Crochet lobular, anticrochet de igual forma pero ligeramente más ancho.

MPG-R-3208 es una porción maxilar que conserva del $\mathrm{P}^{2}$ al $\mathrm{M}^{1}$ en los que se observa un alto grado de desgaste (Figura 5i), que sugiere pertenecieron a un individuo viejo. El $\mathrm{P}^{2}$ sólo está representado por un fragmento del metalofo. En el $\mathrm{P}^{3}$ únicamente se observan remanentes del paracono, metacono, protocono y metalofo. $\mathrm{P}^{4}$ con parastilo prominente; metastilo reducido. Prefoseta apenas visible por el alto grado de desgaste. $\mathrm{M}^{1}$ muy gastado y sólo se diferencia el parastilo.

\subsubsection{Restos apendiculares}

MPG-R-3212 es un metacarpo III izquierdo bien conservado. Longitudinalmente es corto, pero robusto (Figura 6a), rasgo característico en Teleoceras. Los cóndilos lateral y medial son casi del mismo tamaño. La faceta articular para el unciforme es pequeña y forma una prominencia dirigida proximolateralmente. La faceta articular para el magno-trapezoide es plana y casi el doble de tamaño que la faceta articular para el unciforme. La faceta articular para el digito es amplia, tal como se observa en otros perisodáctilos.

MPG-R-3213 es una primera falange del digito III (Figura $6 \mathrm{~b}$ y c). Es casi igual de larga que ancha, por lo que su forma es cuadrangular. El borde del extremo distal es ligeramente cóncavo, mientras que el proximal es casi plano. La faceta que articula con la segunda falange no es muy amplia.

MPG-R-3214 y MPG-R-3215 son magnos derechos (Figura 6d-g), ambos en relativamente buen estado de conservación y sin diferencias significativas en relación a los magnos de Teleoceras previamente descritos. En MPG-R-3215 la faceta articular para el metacarpo II es ligeramente más amplia que la de MPG-R-3214 y el proceso posterior es menos prominente. En ambos especímenes el proceso posterior está bien desarrollado.

\section{Discusión}

Prothero (2005) considera válidas tres especies para el Henfiliano de Norteamérica: T. fossiger (especie de mediano tamaño), presente en el Henfiliano temprano; T. guymonensis (especie de pequeño tamaño) y T. hicksi (especie de mediano tamaño), ambas presentes en el Henfiliano tardío. El material descrito en este trabajo es referido a Teleoceras hicksi con base en la siguiente combinación de caracteres, rinoceronte de mediano tamaño (longitud de las series $\mathrm{M}^{1}-\mathrm{M}^{3}=139 \mathrm{~mm}$, $M_{1}-M_{3}=140 \mathrm{~mm}$ ), nasales cortos que en sección transversal tienen forma de $\mathrm{U}$ y ligeramente orientados hacia arriba, $\mathrm{y}$ ausencia del $\mathrm{P}_{2}$. Aunque las dimensiones de las series dentales $\mathrm{M}^{1}-\mathrm{M}^{3}$ y $\mathrm{M}_{1}-\mathrm{M}_{3}$ de los especímenes provenientes de la Cuenca de Tecolotlán, son menores que los rangos de $T$. hicksi $\left(\mathrm{M}^{1}-\mathrm{M}^{3}=150-165 \mathrm{~mm}, \mathrm{M}_{1}-\mathrm{M}_{3}=155-180 \mathrm{~mm}\right) \mathrm{y}$ caen dentro de las medidas dadas para $T$. guymonensis $\left(\mathrm{M}^{1}-\mathrm{M}^{3}=\right.$ $159 \mathrm{~mm} ; M_{1}-M_{3}=140 \mathrm{~mm}$ ), no se observó en ninguno de los $\mathrm{M}^{3}$ la costilla en la cara lingual del crochet (que forma un pequeño espolón lingual sobre la superficie de desgaste), característico en esta especie (Prothero, 2005). Claramente, el ejemplar MRG-10-295030 era un animal de talla menor a la media establecida para $T$. hicksi. Sin embargo, se descarta la posibilidad de que se trate de una nueva especie, pues la comparación de los ejemplares de la Cuenca de Tecolotlán y los procedentes de la fauna Rancho El Ocote, Guanajuato, determinados como Teleoceras fossiger ( $=$ T. hicksi), no mostró diferencias morfológicas ni de tamaño (Tabla 1) que indiquen se trate de especies distintas. No obstante, se observaron pequeñas diferencias en la morfología craneal y dental que son atribuidas a variación intraespecífica. Por ejemplo, el cráneo MRG-10-295030-A tiene similitudes con el cráneo IGM-8357 del Rancho El Ocote, en la forma 


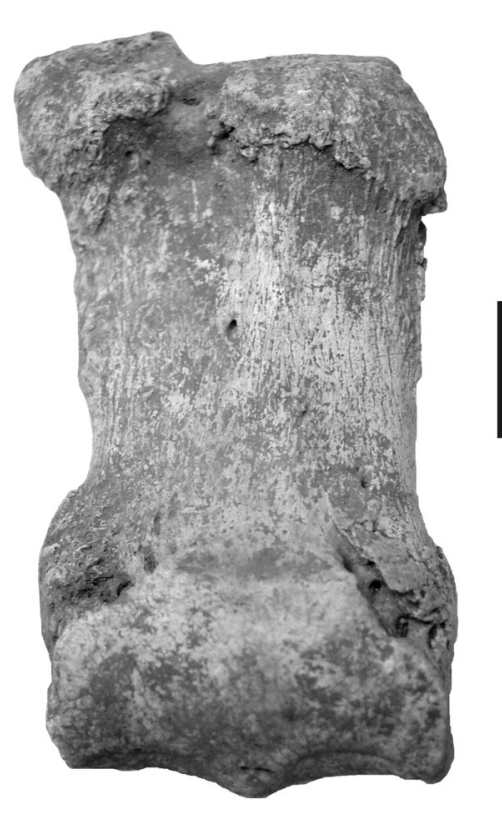

a
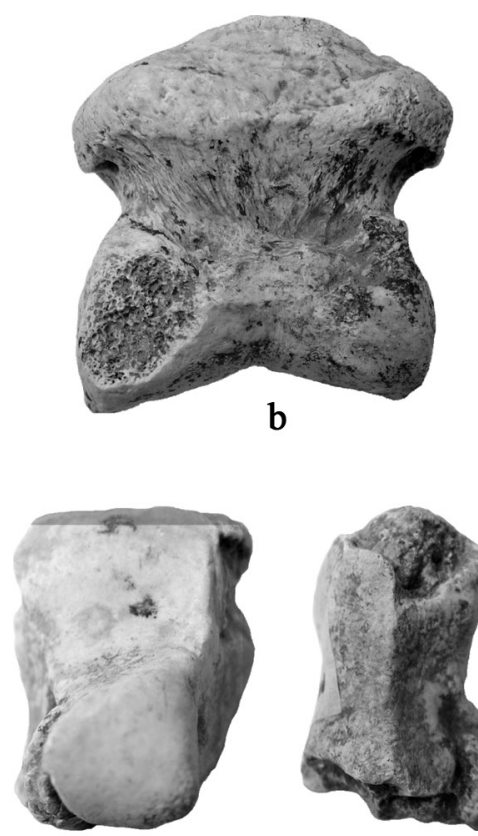

d

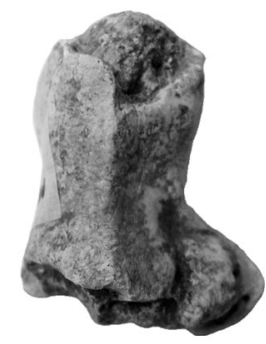

e

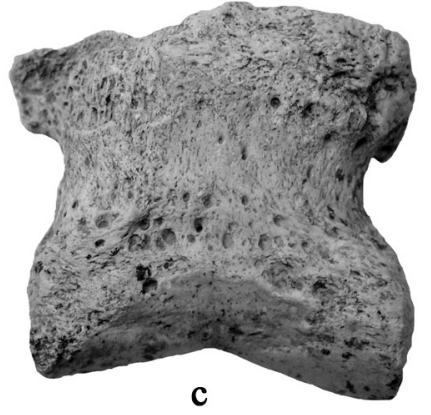

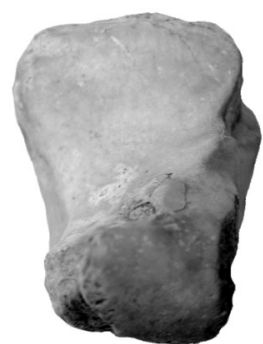

f

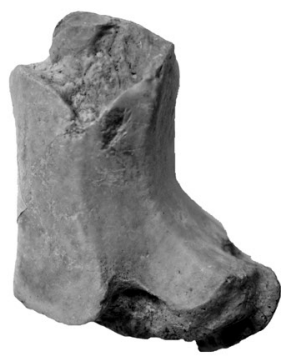

g

Figura 6.- Restos postcraneales de Teleoceras hicksi provenientes de la Cuenca de Tecolotlán, Jalisco: a) MPG-R-3212, metacarpo III izquierdo, vista posterior; b) MPG-R-3213, primera falange, digito III, vista anterior y c) posterior; d) MPG-R-3214, magno derecho, vista proximal y e) distal; f) MPG-R-3215, magno derecho, vista proximal y g) distal. Barra de escala igual a $2 \mathrm{~cm}$.

y constitución de los arcos zigomáticos, apreciándose que en ambos especímenes son más estrechos en su porción anterior que la posterior, mientras que en el cráneo IGM8360 los arcos zigomáticos son casi del mismo ancho en ambos extremos. Las tres mandíbulas descritas en este trabajo no presentan diferencias morfológicas notorias entre sí, ni con el material de Guanajuato, observándose sólo variaciones en el tamaño y constitución de las ramas mandibulares, así como en las dimensiones de los $\mathrm{I}_{2} \mathrm{y}$ de las series $\mathrm{P}_{3}-\mathrm{M}_{3}$ (Tabla 2). En los molariformes la morfología y dimensiones de la superficie oclusal es aun más variada (Tabla 3). Esto se aprecia al observar el M², MPG-R-3204, en donde el crochet es grande y el anticrochet pequeño, al contrario de los $\mathrm{M}^{2}$, MPG-R-3210 y MPG-R-3200, en los cuales el crochet no se observa o es muy pequeño y el anticrochet está muy desarrollado. Algo similar se observa con la forma de la postfoseta de los $\mathrm{M}^{1}$, la cual es constreñida en MRG-10-295030-A, de tal forma que casi se tocan sus paredes interiores por el desarrollo del protolofo y en el $\mathrm{M}^{1}$ MPG-R-3209 es ancha y de forma lenticular. Esta variabilidad sin duda alguna es consecuencia del diferente grado de desgaste presente en la superficie oclusal de cada molariforme, que a su vez es producto del uso, indicando que pertenecieron a individuos de diferente edad. Por el contrario, las diferencias en la constitución craneal y mandibular, pueden ser indicativas de dimorfismo sexual. Carranza-Castañeda (1989) también observó estas variaciones morfológicas tanto en el cráneo, como en los molariformes de los ejemplares que estudió, llegando a la conclusión de que se debían a las diferencias individuales que se presentan en una misma población, las cuales a su vez están influenciadas por la edad y el sexo de cada individuo. Lo anterior también ha sido documentado en Teleoceras fossiger, T. major, T. proterum y Aphelops malacorhinus (Osborn 1898; Mead, 2000; Mihlbachler, 2005). Aunado a las características morfológicas de los especímenes descritos, la edad establecida para la fauna asociada a los restos de rinocerontes de la Cuenca de Tecolotlán es un elemento más que sustenta la asignación del material como T. hicksi.

\section{Conclusiones}

La revisión del material de rinocerontes depositado en el Museo de Paleontología de Guadalajara y en el Museo Regional de Guadalajara, confirma la presencia del rinoceronte Teleoceras hicksi en los sedimentos fluviolacustres de la Cuenca de Tecolotlán, Jalisco. El estudio de los caracteres morfológicos, así como las medidas del material descrito en este trabajo mostraron diferencias mínimas en el cráneo, mandíbulas y principalmente en la superficie oclusal de los molariformes, que se atribuye a la variación intraespecífica que se presenta de forma natural en una población; esto a su vez su vez refleja la presencia de individuos de diferente edad y sexo entre los ejemplares 
estudiados. T. hicksi se encuentra en la Cuenca de Tecolotán asociado con Dinohippus mexicanus, Nannippus aztecus, Neohipparion eurystyle, Astrohippus stockii y Borophagus secundus, por lo que la edad de la fauna corresponde al Henfiliano tardío.

\section{Agradecimientos}

Quisiéramos expresar nuestro agradecimiento a Diana Solórzano Pérez, del Museo de Paleontología de Guadalajara "Federico Solórzano Barreto" y a Martelva Gómez Pineda, del Museo Regional de Guadalajara, por darnos acceso al material de rinocerontes que tienen bajo su resguardo. Ma. del Carmen Perrilliat, encargada de la Colección Nacional de Paleontología, amablemente dio acceso al material de rinocerontes proveniente de El Ocote, Guanajuato. Agradecemos también a Óscar J. Polaco, quien contribuyó con valiosos comentarios en la primera versión del manuscrito, a Óscar Carranza-Castañeda y Esperanza Cerdeño, quienes con sus atinadas sugerencias ayudaron a mejorar este trabajo. G. Carbot-Chanona agradece al proyecto FOMIX CHIS-2005-C03-020 y al Instituto de Historia Natural por financiar la investigación, y a Francisco J. Vega (Instituto de Geología, UNAM) por su valioso apoyo durante el proceso de arbitraje.

\section{Referencias}

Brunet, J., 1968, The Pliocene Rhinoceroses of Mexico (resumen), en Geologic Society of America Annual Meetings, Paper 12, Mexico City, 1-2.

Carbot- Chanona,G., 2008, The first record of Crocodylus (Crocodylomorpha, Crocodylidae) from the Latest Miocene of Chiapas, Mexico, en III Congreso Latinoamericano de Paleontología de Vertebrados, Neuquén, Argentina.

Carranza-Castañeda, O., 1989, Rinocerontes de la Fauna Local Rancho El Ocote, Mioceno tardío (Henfiliano tardío) del estado de Guanajuato: Revista del Instituto de Geología, Universidad Nacional Autónoma de México, 8(1), 88-99.

Carranza-Castañeda, O., 1992, Una nueva localidad del Henfiliano tardío en la Mesa Central de México: Revista del Instituto de Geología, Universidad Nacional Autónoma de México, 10(2), 179-196.

Carranza-Castañeda, O., 2006, Late Tertiary fossil localities in Central Mexico, between $19^{\circ}-23^{\circ} \mathrm{N}$ ", en Carranza-Castañeda, O., Lindsay E.H. (eds), Advances in late Tertiary vertebrate paleontology in Mexico and the Great American Biotic Interchange: Universidad Nacional Autónoma de México, Instituto de Geología y Centro de Geociencias, Publicación Especial, 4, 45-60.

Carranza-Castañeda, O., Ferrusquía-Villafranca, I., 1978, Nuevas investigaciones sobre la fauna Rancho El Ocote, Plioceno medio de Guanajuato, México; informe preliminar: Revista del Instituto de Geología, Universidad Nacional Autónoma de México, 2(2), 163-166.

Castillo-Cerón, J., Cabral-Perdomo, M.A., Carranza-Castañeda,O., 1996, Vertebrados fósiles del Estado de Hidalgo: Universidad Autónoma del Estado de Hidalgo, 127 p.

Cook, H. J., 1927, A new rhinoceros of the genus Teleoceras from Colorado: Proceedings of the Colorado Museum of Natural History, 7, 1-2.

Cook, H.J., 1930, New rhinoceroses from the Pliocene of Colorado and Nebraska: Proceedings of the Colorado Museum of Natural History,
9, 44-51.

Cope, E.D., 1884, The extinct mammalian of the Valley of Mexico: Proceedings of the American Philosophical Society, 22(7), 1-21.

Dalquest, W.W., Mooser, O., 1980. Late Hemphillian mammals of the Ocote local fauna, Guanajuato, Mexico: Texas Memorial Museum, The Pearce-Sellards Series, 32, 1-25.

Ferrusquía-Villafranca, I., 1990, Contribución al conocimiento de los mamíferos miocénicos de Chiapas, México: Revista de la Sociedad Mexicana de Paleontología, 2(2), 77-91.

Freudenberg, W., 1921, Geologie von México: Verlag von Geobrüder Borntraeger, Berlin, 1-32.

Freudenberg, W., 1922, Die säugetierfauna des Pliocäns und Postpliocäns von Mexiko. II Teil, Mastodonten und Elephanten: Geologie und Paläontologie, 14(3), 103-176.

Hatcher, J.B., 1894, A median horned rhinoceros from the Loup Fork beds of Nebraska: American Geologist, 13(3), 149-150.

Kowallis, B.J., Swisher, C.C., Carranza-Castañeda, O., Miller, W.E., Tingey, D.G., 1998, Fision-track and single-crystal ${ }^{40} \mathrm{Ar} /{ }^{39} \mathrm{Ar}$ laserfusion ages from volcanic ash layers in fossil-bearing Pliocene sediments in Central Mexico: Revista Mexicana de Ciencias Geológicas, 15(2), 157-160.

Lance, J.F., 1950, Paleontología y estratigrafía del Plioceno de Yepómera, Estado de Chihuahua, parte I: Equidos, excepto Neohipparion: Boletín del Instituto de Geología, Universidad Nacional Autónoma de México, 5, 87 p.

Lindsay, E.H., 1984, Late Cenozoic mammals from northwestern Mexico: Journal of Vertebrate Paleontology, 4(2), 208-215.

Maldonado-Köerdell, M., 1954, Sobre un rinoceronte fósil de Sinaloa, México: Sociedad Mexicana de Historia Natural, 5(15), 141-146.

Matthew, W.D., 1932, A review of the rhinoceroses with a description of Aphelops material from the Pliocene of Texas: University of California Publications, Bulletin of the Department of Geological Sciences, 20, 411-480.

Mead, A.J., 2000, Sexual dimorphism and paleoecology in Teleoceras, a North American Miocene rhinoceros: Paleobiology, 26(4), 689-706.

Mihlbachler, M.C., 2005, Linking sexual dimophism and sociality in rhinoceroses: insights from Teleoceras proterum and Aphelops malacorhinus from the Late Miocene of Florida: Bulletin of Florida Museum Natural History, 45(4), 495-520.

Miller, W.E., Carranza-Castañeda, O., 1998, The Tecolotlán Basin, Central Mexico: A new area for Late Cenozoic terrestrial vertebrates: Journal of Vertebrate Paleontology, Supplement, 18(3), 26.

Miller, W. E., Carranza-Castañeda, O., 2002, Importance of Mexico's late Tertiary mammalian faunas, en Montellano-Ballesteros, M., ArroyoCabrales, J. (eds.), Avances en los estudios paleomastozoológicos: México, D.F., Instituto Nacional de Antropología e Historia, 83-102.

Montellano-Ballesteros, M., 1997, New vertebrate locality of the late Hemphillian age in Teocaltiche, Jalisco, Mexico: Revista Mexicana de Ciencias Geológicas, 14(1), 84-90.

Osborn, H.F., 1898, A complete skeleton of Teleoceras fossiger. Notes upon the growth and sexual characters of this species: Bulletin of American Museum of Natural History, 10, 51-59.

Osborn, H.F., 1904, New Miocene rhinoceroses with revision of known species: Bulletin of American Museum of Natural History, 20(27), 307-326.

Prothero, D.R., 2005, The Evolution of North American Rhinoceroses: Cambridge University Press, $218 \mathrm{p}$.

Rodríguez-Huerta, M., Guzmán-Gutiérrez, R., 1993, Fauna de vertebrados fósiles del Mioceno tardío del área de Tecolotlán, Jalisco, México, en IV Congreso Nacional de Paleontología: Sociedad Mexicana de Paleontología, 50.

Tanner, L.G., 1975, Stratigraphic occurrences of Teleoceras, with a new Kimballian species from Nebraska: Bulletin of the University of Nebraska State Museum, 10, 23-33.

Manuscrito recibido: Febrero 27, 2009.

Manuscrito corregido recibido: Junio 23, 2009.

Manuscrito aceptado: Junio 28, 2009. 